\title{
Discretization in the quasi-continuum
}

\author{
Ronald S. Burkey and C. D. Cantrell \\ Center for Quantum Electronics, University of Texas at Dallas, P.O. Box 688, Richardson, Texas 75080
}

Received December 1, 1983; accepted January 12, 1984

\begin{abstract}
Straightforward discretization of the equations of motion of a quasi-continuum interacting with an electromagnetic field often leads to physical and numerical difficulties. We derive two distinct methods for reducing the number of energy levels that must be treated explicitly in such calculations. One of these applies to bands of quasi-continua with slowly decreasing shoulders; the other, to bands with rapidly decreasing shoulders.
\end{abstract}

\section{INTRODUCTION}

Beginning with Rice in 1929, many authors have considered a model of the so-called "quasi-continuum" in which a distinguished ground state is coupled to a band of $N$ upper states that are not coupled to one another (see Fig. 1). These efforts are summarized well by Shore. ${ }^{1}$ The $(1, N)$ system allows many analytic results to be derived because it has a simple eigenvalue equation ${ }^{2}$ and because it is susceptible to analysis using the Laplace transform. ${ }^{3-5}$ In the special case (the Rice model) of evenly spaced sublevels of the upper band, Schrödinger's equation can be transformed into a delay-differential equation $^{6,7}$ and hence exhibits recurrences in which the ground-state probability experiences quasi-periodic growth and decay.

In general, previous investigations dealt with the case of the known (simple, classical) form of the electric field acting on a two-level system, or else with a constant electric field. [The case of a sinusoidal field under the rotating-wave approximation (RWA) and the case of a suddenly turned-on field, although physically different from the constant-field case, are mathematically identical to it for times later than the switch-on time.] For an investigation of a laser pulse passing through a medium, ${ }^{7}$ these assumptions about the electric field may not be justified owing to reshaping of the initial pulse as a result of propagation, so it is necessary to consider the case of a time-varying field. Peterson et al. ${ }^{8}$ have studied the adiabatic case, in which the field is turned on slowly rather than suddenly, and have shown that on laboratory time scales even quite fast pulses can sometimes be in the adiabatic rather than the sudden regime.

Yeh et al., ${ }^{\mathbf{4}}$ in contrast to Peterson et al., have recently dealt with the quasi-continuum in the case of a time-varying-field envelope in their "interrupted coarse-graining" theory. They also limit the electric field to be slowly varying. In fact, for the weak-field case their limitation on the pulse turn-on time is just that given in Ref. 8 as a condition for adiabaticity. Thus, for weak fields at least, Yeh's procedure appears to be somewhat akin to the adiabatic approximation; yet it also has features distinct from the adiabatic approximation since continua are not normally considered to possess an adiabatic regime.

Witriol et al. ${ }^{9}$ have considered the problem of reducing the number of levels in a model of a laser-stimulated molecular species reacting to form another species that is removed from the population. This model is, in some ways, more general than the $(1, N)$ model, but the level-reduction scheme is strongly dependent on values of the various system parameters.

We address the same problem as in Ref. 4, namely, that of reducing the difficulty of performing calculations in the (1, $N)$ model of a quasi-continuum with a large number of levels. As in Ref. 9, we reduce a continuum or quasi-continuum of levels to the physically more appealing and computationally cheaper problem of a discrete band with only a few levels. Our techniques are characterized by two properties. First, the field may vary in a totally general way: It may be quantized or classical, and in the latter case the RWA may be applied or not. Second, we strive for high (if not perfect) accuracy rather than merely approximate agreement between the given and reduced systems.

Some of the major questions in laser-induced chemistry in recent years have concerned the nature of intramolecular relaxation (IMR) and the methods by which IMR can be studied experimentally. Recently we pointed out that our preliminary results ${ }^{10}$ for the time dependence of the expectation value of the dipole moment (or other off-diagonal observables) are useful in the context of IMR. Although we do not address the question of IMR in this paper, we intend to do so in a future publication.

\section{INTRODUCTION OF THE THEORY}

Consider a quantum system interacting with an electric field according to the Hamiltonian

$$
H(t)=H_{0}+E(t) \mu,
$$

where the operators $H_{0}$ and $\mu$ are independent of time. If we assume that the RWA has been made, then $H_{0}$ represents a matrix of detunings $\Delta=n \omega-E / \hbar$, rather than a matrix of energies, and $E(t)$ (which absorbs a constant of $1 / 2 \hbar$ ) represents the envelope of the electric field rather than the oscillating field itself. We do not assume that the field envelope is constant. In fact, our main concern is to discover the effects of a nonconstant $E(t)$ on the evolution of the system. Thus the RWA is not forced on us; indeed, we may even allow the field to be quantized. If the RWA is not made we must replace the plus in the definition of $H(t)$ by a minus, or else $\mu$ will represent the negative of the dipole matrix. 


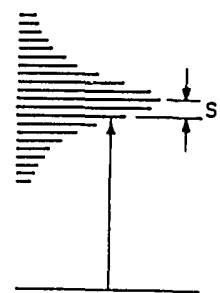

Fig. 1. A typical $(1, N)$ system. Transitions are allowed from the ground state to the upper band, not within the upper band itself.

We concern ourselves here only with the special case in which the system consists of a nondegenerate ground state interacting with a band of upper levels but in which the upper levels are not coupled directly among themselves. As far as the results that we will derive are concerned, the band may be discrete, continuous, or a combination of the two. However, for simplicity, in our discussion and notation we will generally assume that we are dealing with a pure continuum. For such a system we employ the following notation: The ground-state amplitude is denoted by $a(t)$. Upper levels are indexed by the quantity $\Delta=\omega-E / \hbar$, which represents the detuning of the level that it indexes. The probability amplitudes for the upper levels are denoted by $b(t, \Delta)$. The only nonzero elements of the dipole matrix are those coupling the ground state to the upper states, $\mu(\Delta)$, which we assume to be real. For completeness, we also introduce a function $g(\Delta)$, which gives the density of states in the upper band. This allows us to let $\Delta$ vary over the continuous range $(-\infty, \infty)$ even in the discrete case by letting $g(\Delta)$ behave appropriately, e.g., as a delta function in the case of a single discrete level.

The problem that we address is this: Given a system as described above and some simple initial conditions [which we typically choose to be $a\left(t_{0}\right)=1$ and $b\left(t_{0}, \Delta\right)=0$, where $t_{0}$ is a time before which the incident field $E(t)$ vanishes] how can we [for arbitrary fields $E(t)$ ] solve for the time evolution of the system when there may be a large number (possibly an uncountable infinity) of levels in the system? The answer that we envisage for this problem is a reduction of the system in some sense to a system with a much smaller number of levels. In an ideal (and extreme) case we could, perhaps, reduce the number of levels to two or three and solve the system analytically; in a less ideal case we still might be able to reduce the number levels sufficiently to make numerical solutions quite inexpensive.

This, of course, is no new idea. Converting complicated systems to two-level systems (on the grounds that most of the levels do not matter) is not uncommon. However, because of the special system that we have chosen to consider, we try a somewhat different approach to the problem than has been employed in the past.

In our notation, Schrödinger's equations can be written as

$$
\begin{aligned}
\frac{\mathrm{d}}{\mathrm{d} t} a(t) & =i E(t) \int_{-\infty}^{\infty} \mu(\Delta) g(\Delta) b(t, \Delta) \mathrm{d} \Delta, \\
\frac{\mathrm{d}}{\mathrm{d} t} b(t, \Delta) & =i \Delta b(t, \Delta)+i E(t) \mu(\Delta) a(t) .
\end{aligned}
$$

Integrating Eq. (1.2) and substituting into Eq. (1.1) gives the integrodifferential equation

$$
\frac{\mathrm{d}}{\mathrm{d} t} a(t)=-E(t) \int_{t_{0}}^{t} E\left(t^{\prime}\right) a\left(t^{\prime}\right) \chi\left(t-t^{\prime}\right) \mathrm{d} t^{\prime},
$$

where

$$
\chi(t)=\int_{-\infty}^{\infty} \mu(\Delta)^{2} g(\Delta) e^{i \Delta t} \mathrm{~d} \Delta .
$$

All the integrals become sums in the discrete case since the density function becomes a sum of delta functions.

Consider Eq. (1.1) for the time development of $a(t)$, which is the quantity of primary interest given that $b(t, \Delta)$ may be found straightforwardly if $a(t)$ is known. Then it seems natural, in order to discretize Eq. (1.1), to try to replace $b(t$, $\Delta$ ) with some other quantities [in particular, a discrete set of functions $b_{1}(t), b_{2}(t)$, etc.] that have known equations of motion and that give an equation of motion for $a(t)$ but that are not necessarily intuitively related to the actual function $b(t, \Delta)$. In particular, we may suppose that there exist numbers $\Delta_{1}, \Delta_{2}, \Delta_{3}, \ldots$ and $\mu_{1}, \mu_{2}, \ldots$ such that

$$
\begin{aligned}
\frac{\mathrm{d}}{\mathrm{d} t} a(t) & =i E(t) \sum_{n>0} \mu_{n} b_{n}(t), \\
\frac{\mathrm{d}}{\mathrm{d} t} b_{n}(t) & =i \Delta_{n} b_{n}(t)+i E(t) \mu_{n} a(t) .
\end{aligned}
$$

From the similarity of these equations to Eqs. (1.1) and (1.2) and from Eqs. (2) and (3), it is easy to see that the $b_{n}$ will exist if and only if

$$
\chi(t)=\sum_{n>0} \mu_{n}^{2} \exp \left(i \Delta_{n} t\right),
$$

where $\chi(t)$ is as defined earlier. In the sections that follow, we discuss at some length the possibility of finding numbers $\mu_{n}$ and $\Delta_{n}$ for a given system and hence guaranteeing the reduction of Eq. (1.1) to Eq. (4.1). A preliminary discussion of a different but related technique based on tridiagonalization of the Hamiltonian is given in the following section.

Note that we have not assumed in any way that $\mu_{n}$ and $\Delta_{n}$ are real. Thus, even though Eqs. (4.1) and (4.2) superficially resemble Schrödinger's equations, it may be that the system for which these would be Schrödinger's equations would not have a Hermitian Hamiltonian and hence could not exist in the real world. This should not bother us since the $b_{n}$ were introduced merely as a mathematical convenience. However, this realization leads us to ask if the discretized equations of motion (4.1) and (4.2) allow us to compute any quantities of physical interest other than the ground-state amplitude $a(t)$. In fact, they do. The complex polarization $\Phi$, defined ${ }^{7}$ as

$$
\Phi=2 i N \int_{-\infty}^{\infty} a^{*}(t) \mu(\Delta) g(\Delta) b(t, \Delta) \mathrm{d} \Delta,
$$

is easily computed in the discretized system. Multiplying Eq. (1.1) by $2 N a^{*}(t) / E(t)$ is seen to give the complex polarization; on the other hand, substituting for the time derivative of $a(t)$ from Eq. (4.1), we get an expression involving only $a(t)$ and $b_{n}(t)$. Therefore

$$
\Phi=2 i N \sum_{n>0} a^{*}(t) \mu_{n} b_{n}(t) .
$$

It is fortunate that $\Phi$ is so easily calculated in the discretized system, since $\Phi$ is the quantity that couples Schrödinger's equation to Maxwell's equations in the differential equations 
that describe the behavior of an optical field in a bulk material.

From Eqs. (2) and (3) it is clear that the system is completely characterized by the function $\mu(\Delta)^{2} g(\Delta)$, which we call the shape of the band and refer to as $w(\Delta)$ for convenience. We must make a distinction between two cases: whether $w(\Delta) \sim|\Delta|^{-n}$ (for some $n$ ) as $|\Delta| \rightarrow \infty$ or whether $w(\Delta)$ dies away faster than every such power. The reason for this distinction is that we have found methods of dealing with either case but have found no method that can deal with both cases. A deeper reason for the existence of these two disparate cases is not evident.

\section{SLOWLY DYING BANDS}

First, consider the case in which $w(\Delta)$ dies away like $|\Delta|^{-n}$ for some integer $n$ as $|\Delta| \rightarrow \infty$. If this is true, then the function $w(\Delta)$ can be approximated as a rational function, i.e., as a ratio of two polynomials in $\Delta$ :

$$
w(\Delta) \approx \frac{p(\Delta)}{q(\Delta)} \quad[p(\Delta), q(\Delta) \text { polynomials }] .
$$

If such a relation holds, we say that we have a rational band.

Suppose, in fact, that approximation (8) holds strictly rather than approximately. Our main result of this section can be expressed in the following theorem.

\section{Theorem}

If $\mu(\Delta)^{2} g(\Delta)$ is a rational function (with simple poles) of form (8), then a reduction of Eqs. (1) to Eqs. (4) exists. The $\Delta_{n}$ are just the roots of $q(\Delta)$ that lie in the upper half complex $\Delta$ plane, whereas the $\mu_{\mathrm{n}}$ can be expressed by the formula

$$
\mu_{n}=\left[2 \pi i \frac{p\left(\Delta_{n}\right)}{q^{\prime}\left(\Delta_{n}\right)}\right]^{1 / 2},
$$

where the prime on $q(\Delta)$ represents differentiation.

\section{Proof}

Compute $\chi(t)$ according to Eq. (3). $w(\Delta)$ must die away at least as fast as $|\Delta|^{-2}$ or else the total dipole moment [the integral of $w(\Delta)$ ] would not exist. The integral in Eq. (3) can be written, therefore, as the limit of the complex line integral around a large semicircle in the upper half $\Delta$ plane and consequently as a sum of the integrals around the singularities in the upper half-plane (since the integrand is analytic except at those points). Therefore

$$
\chi(t)=\sum_{\Delta_{n}} \exp \left(i \Delta_{n} t\right) p\left(\Delta_{n}\right) \oint_{\Delta_{n}} \frac{d \Delta}{q(\Delta)},
$$

where the sum is to be taken over the upper half-plane roots $\Delta_{n}$ of $q(\Delta)$ and the integrals are evaluated around those points. Since $q(\Delta)$ has no repeated roots, the square of Eq. (9) is the value of the integral as given by complex variable theory. Therefore Eq. (5) is valid (for these values of $\mu_{n}$ and $\Delta_{n}$ ), and so the reduction exists. Q.E.D.

The simplest example of a rational band is the Lorentzian band,

$$
w(\Delta)=\mu(\Delta)^{2} g(\Delta)=\mu^{2} \frac{\sigma}{\pi} \frac{1}{(\Delta-s)^{2}+\sigma^{2}},
$$

where $\sigma$ is the width of the band, $s$ is the offset of the center of the band, and $\mu$ is the total dipole moment. This function has exactly one pole in the upper half $\Delta$ plane, namely, $\Delta=$ $s+i \sigma$, and the application of formula (9) gives the reduced dipole-matrix element $\mu$. Thus the reduced equations of motion for the Lorentzian-band system are

$$
\frac{\mathrm{d}}{\mathrm{d} t}\left[\begin{array}{l}
a(t) \\
b(t)
\end{array}\right]=i\left[\begin{array}{cc}
0 & E(t) \mu \\
E(t) \mu & s+i \sigma
\end{array}\right]\left[\begin{array}{l}
a(t) \\
b(t)
\end{array}\right] .
$$

These equations are often seen as the result of an approximation that is applied when Eq. (2) has been derived but has not been found to be solvable. In fact, these are formally identical with the equations obtained from the WeisskopfWigner approximation. It should be remembered, however, that according to the derivation above they are exact for a Lorentzian band in an arbitrarily varying field. There is no approximation here except possibly the RWA or the approximations inherent in supposing a semiclassical interaction with the electric field. This result has been seen in analytic Lorentzian-band solutions for the case of $E(t)$ constant $^{3}$ and for $E(t)$ exponentially increasing. ${ }^{10}$ Furthermore, it can be applied to give the exact Lorentzian-band solution for any $E(t)$ function for which the two-level solution is known. For example, in Ref. 10 we gave the solution for $a(t)$ for a system of two discrete levels when $E(t)$ is semiexponentially increasing, i.e., when it increases from zero and goes to a constant value in a particular way (see Fig. 2). Now we can immediately generalize that result to obtain $a(t)$ when the upper level is a Lorentzian band. By replacing the detuning in that formula ${ }^{10}$ by the quantity $s+i \sigma$, which includes both the detuning and the bandwidth, we immediately get the desired solution:

$$
a(\tau)=\exp \left(i \zeta_{-} \tau\right) M\left[i \frac{(s+i \sigma) \zeta_{-}}{2 \lambda \alpha},-i \frac{s+i \sigma}{\lambda}, 2 i \alpha \tau\right]
$$

where

$$
\zeta_{-}=\frac{s+i \sigma}{2 \epsilon_{\infty}}-\alpha
$$

and

$$
\alpha=\left[\left(\frac{s+i \sigma}{2 \epsilon_{\infty}}\right)^{2}+\left(\frac{\mu_{01}}{2}\right)^{2}\right]^{1 / 2} .
$$

Here, $M$ is the confluent hypergeometric function.

We conclude this section with a result that is not an application of the rational-band theorem but that is nonetheless

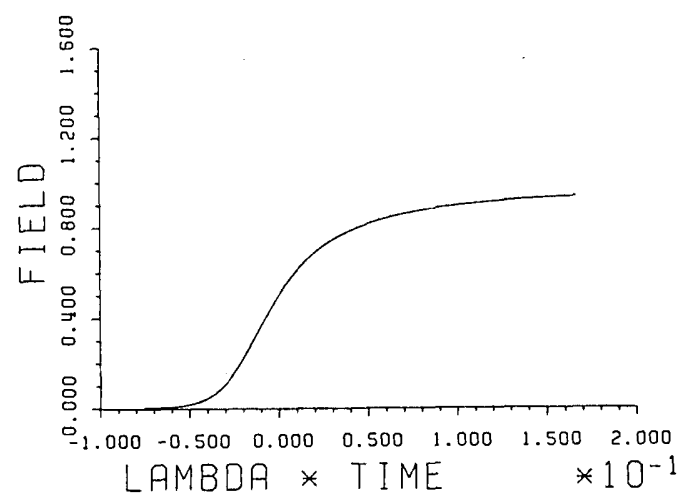

Fig. 2. Form of the semiexponential pulse. Initially $(t=-\infty)$ the field envelope increases as $e^{\lambda t}$, but eventually it goes to a constant value. 
related to the Lorentzian-band result given above. Imagine that, instead of a continuous band of levels, we have an otherwise similar band of evenly spaced discrete levels. In the case of a Lorentzian-band shape, expression (10) must be replaced by

$$
\mu_{n}^{2}=\frac{\sigma \delta}{\pi} \frac{1}{(n \delta-s)^{2}+\sigma^{2}}\left(\tanh \frac{\pi \sigma}{\delta}\right) \mu^{2},
$$

where $\delta$ is the spacing between levels. (In this instance only, $\mu_{n}$ refers to the $n$th level of the given system rather than the reduced system.) If $\chi(t)$ is computed using formula (3), we find $^{3}$ that for $t<2 \pi / \delta$ (the recurrence time of the system)

$$
\begin{aligned}
\chi(t)= & \mu^{2} \frac{1+\tanh \frac{\pi \sigma}{\delta}}{2} \exp [i(s+i \sigma) t] \\
& +\mu^{2} \frac{1-\tanh \frac{\pi \sigma}{\delta}}{2} \exp [i(s-i \sigma) t] .
\end{aligned}
$$

Therefore, so long as we confine our attention to times less than the recurrence time, the discrete Lorentzian system can be reduced exactly to a three-level system with detunings $s$ $\pm i \sigma$ and dipole-matrix elements given by the square roots of the coefficients of the exponentials in the equation above. If we compare this with the result seen in the case of a continuous Lorentzian band, we will notice that this three-level reduction is equivalent to replacing the discrete Lorentzian band by two continuous Lorentzian bands. Mathematically, one of the continuous bands has width $\sigma$ and the other has the negative width $-\sigma$. Presumably it is this negative-width band that causes the breakdown of the reduction at the first recurrence. When $\tanh (\pi \sigma / \delta) \approx 1$, the negative-width band is almost decoupled from the rest of the system, and only the positive-width band remains. This is reasonable since when the spacing $\delta$ is less than the bandwidth $\sigma$ we might expect that the levels in the discrete Lorentzian would be dense enough to justify a continuum approximation.

\section{QUICKLY DYING BANDS}

Consider the case in which $w(\Delta)$ dies away as $|\Delta| \rightarrow \infty$ faster than $|\Delta|^{-n}$ for any $n$. If this is true, then it is possible to define orthogonal polynomials $f_{n}(\Delta)$ with respect to the weight function $w(\Delta)$ :

$$
\int_{-\infty}^{\infty} w(\Delta) f_{n}(\Delta) f_{m}(\Delta) \mathrm{d} \Delta=\delta_{n m}
$$

Given that it is possible to define orthogonal polynomials, there is a systematic procedure for developing approximation formulas for the class of integrals

$$
\int w(\Delta) f(\Delta) \mathrm{d} \Delta
$$

where $f(\Delta)$ is to be an arbitrary function as far as the formula is concerned. ${ }^{11,12}$ Furthermore, there is a systematic procedure for deriving the error terms of such formulas. ${ }^{13}$ In general, one finds a linear-approximation formula of the form

$$
\int_{-\infty}^{\infty} w(\Delta) f(\Delta) \mathrm{d} \Delta \approx \sum_{n>0} w_{n} f\left(\Delta_{n}\right)
$$

where the $\Delta_{n}$ are the roots of one of the orthogonal polyno- mials $f_{n}(\Delta)$ and the $w_{n}$ are weight factors. The error term (which is not shown) involves a high-order derivative of $f(\Delta)$. If we apply approximation (12) to the case $f(\Delta)=e^{i \Delta t}$, we get

$$
\chi(\mathrm{t}) \approx \sum_{n>0} w_{n} \exp \left(i \Delta_{n} t\right),
$$

which is of the form of Eq. (5). Consequently, a quickly dying band can be approximately discretized by putting levels at the positions of the roots of one of the orthogonal polynomials and using as dipole-matrix elements the square roots of the weight factors that would be needed for an approximate integration formula based on the orthogonal polynomial.

The error in a calculation of the ground-state probability amplitude using such a scheme can be approximated by applying mean-value integral formulas to integrodifferential Eq. (2). If $E(t)$ does not become too large within the decay time of $\chi(t)$, it can be shown that the maximum error in the ground-state amplitude on the time interval $[0, t]$ is less than

$$
\delta \chi \int_{0}^{t} \int_{0}^{t^{\prime}} E\left(t^{\prime}\right) E\left(t^{\prime \prime}\right) \mathrm{d} t^{\prime \prime} \mathrm{d} t^{\prime}
$$

where $\delta \chi$ is the maximum error in $\chi(t)$ on this interval. The units in this expression are correct if we recall that $E(t)$ is taken to include a factor of $1 / 2 \hbar$.

A more obvious way to discretize a continuous band would be simply to replace the continuum by a set of evenly spaced levels with dipole-matrix elements that are roughly the same as (or proportional to) the value of the $\mu(\Delta)$ at the corresponding position. (For convenience, we refer to this procedure as Rice discretization since equally spaced levels are involved.) Rice discretization is not the best choice for precisely the same reason that choosing equally spaced sample points in approximation formula (12) is not the best choice. There is also a physical reason for avoiding Rice discretization. Equally spaced levels cause the phenomenon of recurrences, in which constructive and destructive interference create a quasi-periodic oscillation in the ground-state amplitude. We saw earlier in the case of the discrete Lorentzian band that the reduction to a three-level system worked until the first recurrence time, but then it broke down. The approach of using expression (13) as a prescription for discretization avoids this trap and optimizes the discretization at the same time.

Expression (14) for the error in $a(t)$ that is due to the error in $\chi(t)$ implies that our version of the discretization also breaks down eventually. In fact, since the error term of expression (13) involves a derivative of $f(\Delta)$ (which is $e^{i \Delta t}$ ), the error in $\chi(t)$ must have a polynomial dependence on $t$. Thus the reduction does fail in time, but the error expression gives us a means to estimate the time range for which the approximation is valid.

As an example, consider a uniform continuous band of width $2 \Delta_{0}$ in which $w(\Delta)$ is $\mu^{2} /\left(2 \Delta_{0}\right)$ for $|\Delta|<\Delta_{0}$ and is zero outside this range. For this band shape, the orthogonal polynomials are Legendre polynomials. The discretized system therefore has a ground state and a band of discrete levels positioned according to the roots (times $\Delta_{0}$ ) of a Legendre polynomial; the dipole-matrix elements are proportional (by a factor of $\mu / \sqrt{2}$ ) to the square roots of the weight factors for Gaussian integration and connect the levels in the 
band to the ground state but not to other levels in the band. The error formula for Gaussian integration ${ }^{11}$ guarantees a bound on the error in $\chi(t)$. If there are to be $N$ levels in the discretized band, the error in $\chi(t)$ is given by

$$
|\delta \chi| \leq \frac{2^{2 N+1}(N !)^{4}}{(2 N+1)[(2 N) !]^{3}}\left(\Delta_{0} \mathrm{t}\right)^{2 \mathrm{~N}}
$$

As a numerical example, take the case $\Delta_{0}=0.3 \mathrm{~cm}^{-1}$, with $\mu E(t)$ ramping linearly from zero to $0.05 \mathrm{~cm}^{-1}$ at $t=1 / 30 \mathrm{nsec}$ and remaining constant thereafter. We adopt the goal of providing six-figure accuracy for the ground-state probability amplitude on the time interval $t<1 / 3$ nsec. As may be expected, expression (14) actually gives a slightly pessimistic estimate for the necessary number of discretized levels. We have integrated Schrödinger's equation using 16 discretized levels chosen as discussed above. The resulting ground-state probability amplitude (which is real) is depicted in Fig. 3. In contrast, consider the Rice discretization, which involves replacing the band by a set of evenly spaced discrete levels connected to the ground state by equal dipole-matrix elements. Figure 4 displays the error in the ground-state probability amplitude calculated from Rice discretizations with $16,32,64$, and 128 evenly spaced levels, compared with the "correct" ground-state amplitude calculated from the system as discretized by our method described above (using 16 levels). Among the Rice discretizations, only the 128-level case attains six-figure accuracy. In Fig. 4, notice that the ground-state probability amplitudes as obtained from the Rice discretizations appear to coincide periodically (and simultaneously) with the true ground-state amplitude. The reason for this novel feature is not entirely clear to us.

Although the technique of approximating $\chi(t)$ using orthogonal-polynomial methods of quadrature obviously leads to quite satisfactory results compared with simpler discretization schemes, the application of formula (13) has two inconvenient aspects. First, and most important, we have to have an effective method of computing the roots of the orthogonal polynomials and a method of computing the weight factors. Second, we cannot build a more accurate discretization from a less accurate one. That is, if we decide that we must have more accuracy, then there is no alternative to using an orthogonal polynomial of higher degree, computing its

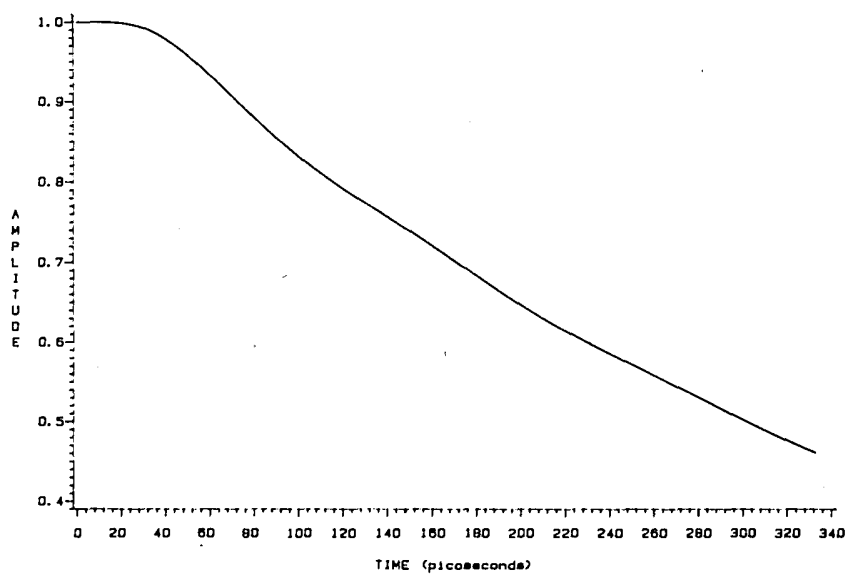

Fig. 3. Ground-state probability amplitude for a uniform rectangular band of full width $0.6 \mathrm{~cm}^{-1}$, as calculated by our technique using 16 levels in the discretized band. This result is accurate to about six decimal places.

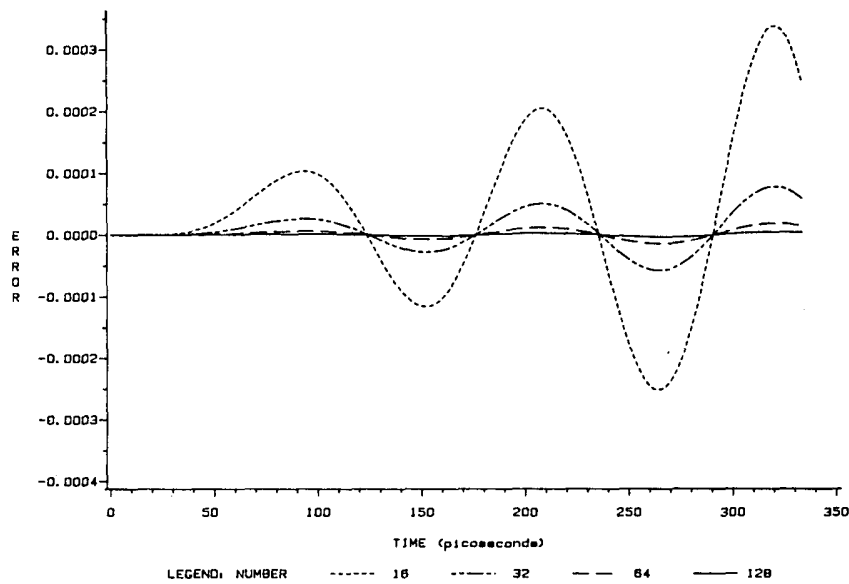

Fig. 4. Error resulting from Rice discretizations of 16, 32, 64, and 128 evenly spaced levels in the calculation of the of full width $0.6 \mathrm{~cm}^{-1}$. The zero point of errornd is taken to be the amplitude calculated using our method (see Fig. 3).

roots, etc. We cannot simply take the less accurate discretization, add a few more levels, and try again.

This is all that we have to say on discretization within the framework of Eq. (5). As it happens, however, there is another approach to the discretization of quickly dying bands that, although it is based on a completely different premise about how discretization should be performed, uses similar mathematical machinery to that discussed above, and in a more convenient way.

The alternative approach to discretization can be summed up in the following theorem.

\section{Theorem}

The Hamiltonian of any system (of the type that we are discussing in this section) can be tridiagonalized by a similarity transformation that is independent of $E(t)$. The diagonal elements of the Hamiltonian are

$$
0, \quad-\frac{d_{0}}{e_{0}}, \quad-\frac{d_{1}}{e_{1}}, \quad-\frac{d_{2}}{e_{2}}, \ldots,
$$

and the codiagonal elements are

$$
E(t) \mu, \quad \frac{1}{e_{0}}, \quad \frac{1}{e_{1}}, \quad \frac{1}{e_{2}}, \ldots,
$$

where the $d_{n}$ and $e_{n}$ come from a recurrence formula ${ }^{12}$ of the orthogonal polynomials $f_{n}$ with respect to $w(\Delta)$,

$$
f_{n+1}(\Delta)=\left(d_{n}+e_{n} \Delta\right) f_{n}(\Delta)-c_{n} f_{n-1}(\Delta) \text {. }
$$

\section{Proof}

We explicitly give the similarity transformation. Note that, although our vectors may have many elements, we represent them as having just two components, a scalar and a function of $\Delta$; the scalar represents the ground-state component, and the function represents the upper-level components. With this notation, the new basis vectors are

$$
\left[\begin{array}{l}
1 \\
0
\end{array}\right], \quad\left[\begin{array}{c}
0 \\
\frac{\mu(\Delta)}{\mu} f_{0}(\Delta)
\end{array}\right], \quad\left[\begin{array}{c}
0 \\
\frac{\mu(\Delta)}{\mu} f_{1}(\Delta)
\end{array}\right], \ldots
$$

Given the exact form of these vectors, the Hamiltonian matrix 
is easily seen to be as stated in the theorem. That the vectors are orthogonal and form a complete set is a trivial consequence of the orthogonality and completeness of the orthogonal polynomials. Q.E.D.

This theorem puts us in a much better position than that in which we were left by the previous method since, even though we still need to know the orthogonal polynomials, we no longer need to know the roots and weight factors. Further, successively more accurate discretizations can now be built up recursively.

The manner in which the total dipole strength and the band shape figure independently in the tridiagonal Hamiltonian is astonishing. Only two matrix elements depend on $E(t)$ and $\mu$. All other nonzero elements in the matrix are somehow geometric constants that depend only on the shape (and not on the strength) of the band. To compute the complex polarization we need only the first two probability amplitudes. Hence any approximation that we make regarding the elimination of the higher levels affects the polarization (which is the only physical quantity other than the population that interests us) only indirectly.

If we were to truncate the Hamiltonian matrix, keeping just the low levels, we would find that the remaining matrix is actually similar to the discretized Hamiltonian derived earlier in this section, in which the detunings are the roots of some orthogonal polynomial and the dipole-matrix elements are the square roots of the weight factors of an approximate integration scheme based on the orthogonal polynomial. This is perhaps not surprising since the mathematics of orthogonal polynomials permeates the entire subject. Nevertheless, this observation serves to connect what we have found here to the general framework of discretization embodied in Eq. (5). Furthermore, since the previous method of discretization in this section permitted the derivation of error formulas, we can obtain estimates from these formulas of the error involved in truncating the tridiagonal Hamiltonian.

The notion that the mathematics of orthogonal polynomials may be useful in solving the dynamics of quantum systems, particularly in the tridiagonal case, is not new.,14,15 Orthogonal polynomials have generally been seen as a tool for computing the dressed-state eigenvalues and eigenvectors of a system experiencing a constant-amplitude field (or perhaps a field that has been suddenly switched on). What we have found is valid for a field varying in an entirely general way.

References 7 and 14 nevertheless present results relevant to the tridiagonal method of discretization. These authors start with a tridiagonal Hamiltonian (which is truncated for the sake of computer calculations) and solve (in the case of a constant field) for the populations. The matrix elements of the Hamiltonian are chosen as recursion coefficients for the Chebyshev, Hermite, Legendre, or Laguerre orthogonal polynomials. Thus, in light of the theorem given above, we see that the numerical results given in Ref. 14 can be viewed as approximations (in the case of a constant field) to populations in systems containing continuous bands of various shapes-among which are included the Gaussian band shape and the uniform rectangular band shape.

We do not discuss the tridiagonal method of discretization further here. In a future publication, we will show that the method can be vastly generalized. The detailed discussion of the method is more appropriate in that context than in this.

\section{SUMMARY AND DISCUSSION}

We have considered the discretization of systems in which there is a ground state and a band of levels that interact with the ground states but not with each other. This band is envisaged as a continuum, and the problem is to introduce in place of the band a discrete set (it is hoped that the set will be finite) of quantities that can be used to calculate the ground-state amplitude and the complex polarization. This is not the same problem as computing the eigenvalues and eigenvectors since we want our discretization to be useful in the case of a time-varying electric field $E(t)$. In fact, only reductions that are good in the case of a totally general $E(t)$ have been discussed, and hence the results derived can be coupled with a numerical-differential-equation solver to solve Schrödinger's equation for the type of system that we have considered.

We have found two essentially separate cases. In the one case, in which $w(\Delta)=\mu(\Delta)^{2} g(\Delta) \sim|\Delta|^{-n}$ (for some integer $n)$ as $|\Delta| \rightarrow \infty$, we have found that $w(\Delta)$ can be approximated as a rational function $p(\Delta) / q(\Delta)$. We have shown how to find $N$ quantities [where the degree of $q(\Delta)$ is $2 N$ ] for which we know the equations of motion and in terms of which we can express the equation of motion of the ground-state amplitude $a(t)$. These equations of motion resemble those for an $(N+$ 1)-level Schrödinger equation, except that the Hamiltonian need not be Hermitian. There is no approximation in these equations beyond the rational approximation, and the ground-state amplitude and complex polarization can be computed [for arbitrary fields $E(t)$ ] after arbitrarily long times in the reduced system.

In the second case, where $w(\Delta)$ dies to zero faster than any power of $|\Delta|$ as $|\Delta| \rightarrow \infty$, we have found a similarity transformation that tridiagonalizes the Hamiltonian. The tridiagonal Hamiltonian is discrete but infinite, and the only approximation involved is in the truncation of this matrix to give a finite system. The similarity transformation is completely independent of time, so that once again the reduced system can be used in the case of a time-varying field. Furthermore, the only elements of the Hamiltonian matrix that depend on the field connect the ground state with the next higher level. Thus only these two levels have to be accurately known to compute the complex polarization. Since any truncation of the Hamiltonian (or, possibly, replacement of the upper states by a reservoir) will leave the equations of motion of these levels unchanged (though altering the actual values of some of the probability amplitudes entering into them), the effect of such an approximation on the ground-state amplitude or the complex polarization can only be indirect. We have also given the matrix obtained from a certain similarity transformation of the truncated tridiagonal Hamiltonian and shown how it can be used to give an expression for the error involved in the truncation.

\section{ACKNOWLEDGMENTS}

We thank Daniel R. Adams and C. B. Collins for help in producing Figs. 3 and 4 and R. E. Wyatt for drawing our attention to the work of Roger Haydock.

This research was supported by the Robert A. Welch Foundation under grant AT-873. 
Note Added in Proof. Witriol ${ }^{16}$ advocates direct numerical solution of integrodifferential equation (2). This a form of coarse graining in the time domain. We do not mean to imply that we have a method of attack for every possible case. If, for example, one shoulder dies as $|\Delta|^{-n}$ while the other dies as $|\Delta|^{-m}$ with $n>m$, then Eq. (8) is invalid and the approach that follows it fails.

\section{REFERENCES}

1. B. W. Shore, "Coherence in the quasi-continuum model," Chem. Phys. Lett. 99, 240-243 (1983).

2. J. H. Wilkinson, The Algebraic Eigenvalue Problem (Oxford U. Press, Oxford, 1965); E. U. Condon and G. H. Shortley, The Theory of Atomic Spectra (Cambridge U. Press, Cambridge, 1935), pp. 40-41.

3. A. A. Makarov, V. T. Platonenko, and V. V. Tyakht, "Interaction of a 'level-band' quantum system with a quasiresonant monochromatic field," Zh. Eksp. Teor. Fiz. 75, 2075-2091 (1978) [Sov. Phys. JETP 48, 1044-1051 (1978)].

4. J. J. Yeh, C. M. Bowden, and J. H. Eberly, "Interrupted coarsegrained theory of unimolecular relaxation and stimulated recurrences in photo-excitation of a quasi-continuum," J. Chem. Phys. 76, 5936-5946 (1982); see also J. H. Eberly, J. J. Yeh, and C. M. Bowden, "Interrupted coarse-grained theory of quasicontinuum photo-excitation," Chem. Phys. Lett. 86, 76-80 (1982).

5. G. C. Stey and R. W. Gibberd, "Decay of quantum states in some exactly soluble models," Physica 60, 1-26 (1972).

6. P. W. Milonni, J. R. Ackerhalt, H. W. Galbraith, and M.-L. Shih, "Exponential decay, recurrences, and quantum-mechanical spreading in a quasi-continuum model," Phys. Rev. A 28, 32-39
(1983); R. Lefebvre and J. Savolainen, "Memory functions and recurrences in intramolecular processes," J. Chem. Phys. 60, 2509-2555 (1974).

7. C. D. Cantrell, V. S. Letokhov, and A. A. Makarov, "Coherent excitation of multilevel systems by laser light" in Coherent Nonlinear Optics: Recent Advances, M. S. Feld and V. S. Letokhov, eds. (Springer-Verlag, Berlin, 1980).

8. G. L. Peterson, C. D. Cantrell, and R. S. Burkey, "Adiabatic excitation of multilevel band systems," Opt. Commun. 43, 123-127 (1982).

9. N. M. Witriol, A. J. Galli, W. H. Brumage, and C. M. Bowden, "Criteria for the reduction of the effective manifold of states in models of laser-induced dissociation and chemistry," Opt. Lett. 5, 24-26 (1980).

10. R. S. Burkey and C. D. Cantrell, "Solution of the Schrödinger equation for systems driven by an exponential or a semiexponential pulse," Opt. Commun. 43, 64-68 (1982).

11. U. W. Hochstrasser, "Orthogonal polynomials" in Handbook of Mathematical Functions, M. Abramowitz and I. A. Stegun, eds. (U.S. Government Printing Office, Washington, D.C., 1964), p. 773.

12. D. Jackson, Fourier Series and Orthogonal Polynomials (Collegiate, Menasha, Wisc., 1941), Chap. 7.

13. R. W. Hamming, Numerical Methods for Scientists and Engineers (McGraw-Hill, New York 1962), pp. 118-164.

14. R. Haydock, "The recursive solution of the Schrödinger equation," Comput. Phys. Commun. 20, 11-16 (1980); R. Haydock, "The recursive solution of the Schrödinger equation," in Solid State Physics, H. Ehrenreich, F. Seitz, and D. Turnbull, eds. (Academic, New York, 1980), Vol. 35, p. 215.

15. Z. Bialynicka-Birula, I. Bialynicka-Birula, J. H. Eberly, and B. W. Shore, "Coherent dynamics of $N$-level atoms and molecules. II. Analytic solutions," Phys. Rev. A 16, 2048-2051 (1977).

16. N. M. Witriol, "Including the continuum in the $N$-level molecule model," Chem. Phys. Lett. 98, 77-80 (1983). 